\title{
Key health themes and reporting of numerical cigarette-waterpipe equivalence in online news articles reporting on waterpipe tobacco smoking: a content analysis
}

\author{
Mohammed Jawad, ${ }^{1}$ Ali M Bakir, ${ }^{2}$ Mohammed Ali, $^{2}$ Sena Jawad, ${ }^{3}$ Elie A Akl ${ }^{4,5,6}$
}

- Additional material is

published online only. To view please visit the journal online (http://dx.doi.org/10.1136/ tobaccocontrol-2013-050981)

1 Department of Primary Care and Public Health, Imperial College London, London, UK ${ }^{2}$ Faculty of Medicine, Imperial College London, London, UK

${ }^{3}$ Department of Medical Statistics, London School of Hygiene and Tropical Medicine, London, UK

${ }^{4}$ Department of Internal Medicine, American University of Beirut, Beirut, Lebanon

${ }^{5}$ Department of Clinical Epidemiology and Biostatistics, McMaster University, Hamilton, Canada

${ }^{6}$ Department of Medicine, State University of New York at Buffalo, Buffalo, New York, USA

\section{Correspondence to} Dr Mohammed Jawad, Department of Primary Care and Public Health, Imperial

College London, London W6 8RP, UK:

mohammed.jawad06@

imperial.ac.uk

Received 21 January 2013 Accepted 30 June 2013 Published Online First 18 July 2013

\section{ABSTRACT \\ Introduction There is anecdotal evidence that health} messages interpreted from waterpipe tobacco smoking (WTS) research are inconsistent, such as comparing the health effects of one WTS session with that of 100 cigarettes. This study aimed to identify key health themes about WTS discussed by online news media, and how numerical cigarette-waterpipe equivalence (CWE) was being interpreted.

Methods We identified 1065 online news articles published between March 2011 and September 2012 using the 'Google Alerts' service. We screened for health themes, assessed statements mentioning CWE and reported differences between countries. We used logistic regression to identify factors associated with articles incorrectly reporting a CWE equal to or greater than 100 cigarettes, in the absence of any comparative parameter ('CWE $\geq 100$ cigarettes').

Results Commonly mentioned health themes were the presence of tobacco (67\%) and being as bad as cigarettes (49\%), and we report on differences between countries. While $10.8 \%$ of all news articles contained at least one positive health theme, $22.9 \%$ contained a statement about a CWE. Most of these (18.6\% total) were incorrectly a CWE $\geq 100$ cigarettes, a quarter of which were made by healthcare professionals/ organisations. Compared with the Middle East, articles from the USA and the UK were the most significant predictors to contain a CWE $\geq 100$ cigarettes statement. Conclusions Those wishing to write or publish information related to WTS may wish to avoid comparing WTS to cigarettes using numerical values as this is a major source of confusion. Future research is needed to address the impact of the media on the attitudes, initiation and cessation rates of waterpipe smokers.

\section{INTRODUCTION}

Waterpipe tobacco smoking (WTS), also known as shisha, hookah and narghile, is prevalent across the world. ${ }^{1}$ Despite growing knowledge that its health effects may be analogous to those of cigarette use, ${ }^{2-4}$ there exists a common perception that it is safer than cigarettes. ${ }^{5}$ Public health organisations have ascertained that WTS is a threat to public health. $^{7}$

Article 12 of the WHO Framework Convention on Tobacco Control (FCTC) identifies communication of the health risks of smoking as a key aspect of tobacco control. ${ }^{8}$ The primary focus of the framework is the use of cigarettes. There has been little attention paid to the use of waterpipes, and in particular, the regulation of packaging, health warnings and descriptors. In Lebanon, for example, the surface area of health warnings average only $3.5 \%$, and packages contain misleading descriptors, such as ' $0 \%$ tar' and ' $0.5 \%$ nicotine', contrary to FCTC requirements.'

WTS research is still in its infancy. A smoking machine has identified that smokers may inhale 100 cigarettes' worth of smoke, whose content roughly equates to 50 cigarettes' worth of tar, 10 cigarettes' worth of carbon monoxide and 2 cigarettes' worth of nicotine per 45 min session. ${ }^{10}$ WTS is smoked differently to cigarettes. For example, it is smoked over a long period of time (usually over $45 \mathrm{~min}$ ), with up to 100 puffs per session (each puff deeper than that seen by cigarette users) and in a social setting, often shared with peers. ${ }^{10}$ As it is also smoked intermittently, the health effects resulting from its use may differ from cigarettes. In addition to nicotine, dependence on waterpipe may be constructed by strong sensory cues (aromatic smell, honey/fruit flavoured taste), peer influences and widespread social acceptance. ${ }^{11}$ These are fundamental differences that health promoters must appreciate when engaging with their target audience, yet there are reports that compare one WTS session to 100 cigarettes $^{12}{ }^{13}$ or more ${ }^{14}$ without mentioning the volume of smoke. This gives the impression to the reader that WTS is 100 times worse than cigarettes, when in fact it is only the volume of smoke produced which is equivalent to the volume of smoke produced from 100 cigarettes. This has caused an array of confusion when communicating the health effects of WTS, though the degree to which this confusion is present is currently uncertain.

Public health agents frequently use news media for communicating health risks to the general public. There is evidence that heightened media coverage of cigarette smoking can modify the population rates of smoking cessation ${ }^{15}{ }^{16}$ and attitudes towards smoking, ${ }^{17}$ and may be as effective as a $10 \%$ price increase. ${ }^{18}$ Research from Australia revealed that an adult is exposed to tobacco news issues approximately once every few weeks. ${ }^{19}$

The internet is also being increasingly used as a source of news and health information. Consequently, online news media may be playing an active role in shaping the attitudes towards WTS as has been noted for cigarette smoking. Therefore the aim of this study was to explore how different countries described waterpipe health themes, and 
whether they included a comparison of one WTS session to 100 cigarettes without mentioning volume of smoke or other parameters, or whether they included any other numerical cigarettewaterpipe equivalence (CWE) measure.

\section{METHODS}

\section{Eligibility criteria}

We included all 'original' online news articles mentioning either positive or negative health themes associated with WTS, including herbal variants. Different online news sources publish identical news articles and thus 'original' refers to only the news article that was published earliest. We excluded legislative news articles, unless health risks were specifically mentioned. We also excluded secondary news articles, blogs, discussions, books, news articles with no publication date, news articles accessible only through paid subscription, and those that did not clearly differentiate the health effects of WTS from other tobacco products.

\section{Search strategy}

We used the internet content monitoring service 'Google Alerts' to receive news articles in English by email using keywords related to WTS, such as 'waterpipe', 'shisha', 'hookah', 'narghile' and 'hubble-bubble' (see online supplementary appendix 1). 'Google Alerts' functions by automatically and regularly searching the internet for new content relating to specific keywords, and can be tailored to focus solely on news articles (http://www.google.com/alerts). We decided to run the service until approximately 1000 eligible articles were received. This took about 18 months (3 March 2011-14 September 2012). We also searched secondary online tobacco news sources (see online supplementary appendix 2) to find other eligible news articles within the same time period.

\section{Selection process}

One reviewer screened for potential eligibility of news articles by reading the news headline provided by each 'Google Alerts' email, and the short excerpt of the news article it provided. We noted eligible news articles on a pilot-tested spreadsheet and removed duplicates.

\section{Data abstraction}

Two investigators independently abstracted data using a pilottested spreadsheet. They resolved any discordance by discussion or consulting a third reviewer. After reviewing the first 200 news articles, inter-reviewer agreement produced a Cohen's $\kappa$ result of $0.67,{ }^{20}$ so the abstraction process was continued by one investigator. We abstracted data using three broad categories. The first theme was descriptive features about the article, such as the date of publication and the presence of direct/indirect citation of scientific research (Yes/No). Our second theme centred around social media, abstracting data on the presence and number of Twitter 'retweets' and Facebook 'likes' (Yes/No) and the presence of video or audio (Yes/No). Our final theme focused on the type of health theme mentioned, such as diseases, chemical content of waterpipe tobacco, dispelling myths and CWE. After the analysis, these variables were coded into similar concepts, for example, WTS contains nicotine or causes addiction.

We paid particular attention to coding news articles in which a numerical CWE was present. Using data from waterpipe smoke machines and plasma levels of smokers, we performed a literature review on the chemical content of smoke aerosol produced from one WTS session to estimate acceptable ranges for comparing different toxicants of waterpipe and cigarettes. We used the following estimates: one WTS session may produce 22-50 times more 'tar' than a single cigarette, ${ }^{21}{ }^{22}$ 6-13 times more carbon monoxide, ${ }^{22} 231-10$ times more nicotine. ${ }^{22}{ }^{24} \mathrm{We}$ also noted that the volume of smoke produced by one waterpipe session may equal 50-250 times the volume of smoke produced by one cigarette. ${ }^{21} 25$ Should any online news article mention a numerical CWE, the investigator would note the parameter (chemical type or volume of smoke, if applicable) which was being compared. If no parameters were present, the CWE was considered to be a 'direct numerical comparison to cigarettes'; for example, one news article headline read: 'Shisha the same as 100 fags.'

We corroborated our abstraction on social media information by running a computer program to automatically download such data on each news article. This program also downloaded popularity statistics for each news article calculated by number of visitors and URL links leading to the website, which gave each website a popularity ranking (known as the 'Alexa rank'). Additionally, the program gave us information on the country source of each news article, which we grouped into the top five most popular countries in addition to a smaller, 'Other' group for remaining countries. News articles in the 'Other' group were grouped into larger regions (Rest of Europe, Africa, Middle East, East Asia, Other), but only news articles from the Middle East were large enough in frequency to enable a categorical group for comparison. This resulted in categorising country source into Middle East, USA, UK, Canada, India, Pakistan and Other.

\section{Data analysis}

We ran descriptive statistics of the collected data using percentages for categorical values and mean and SD for continuous variables. Also, we analysed the publication frequency of news articles to identify and explain clusters of media reporting using a 3-day moving average. Due to the high frequency of incorrect numerical CWEs which compared one WTS session to 100 cigarettes or more in the absence of any other parameters of comparison (ie, chemical content or volume of smoke), the authors felt this warranted further investigation. We therefore performed backward stepwise logistic regression on online articles that contained this type of numerical CWE. We calculated adjusted ORs with 95\% CIs. Our predictor variables were country/region source (Middle East/USA/UK/Canada/India/ Pakistan/Other), website popularity (each website was numerically ranked according to visitor popularity and number of URL links that lead to the website, as identified automatically by the 'Alexa rank'), and direct/indirect citation of scientific research (Yes/No). We analysed data using Statistical Package for the Social Sciences (SPSS) V.17.0.

\section{RESULTS}

The 'Google Alerts' service identified 1065 unique news articles mentioning WTS health themes (1.9 news articles/day), all of which were found on secondary tobacco news websites within the same time period (see online supplementary appendix 2). Most news articles originated from India (22.4\%), Pakistan $(19.0 \%)$ and the USA (18.8\%) (table 1). The vast majority of news article sources were social media enabled $(91.3 \%)$ and $3.9 \%$ contained video or audio material; $11.3 \%$ were from websites ranked within the top 999 websites online according to their 'Alexa rank' and a further 29.3\% were ranked between 1000 and 9999 of all websites. Most news articles were retweeted by its readers, but only $8.5 \%$ had 15 or more 
Table 1 Country/region of origin of all news articles $(n=1065)$

\begin{tabular}{lrr}
\hline Country or region & N & $\%$ \\
\hline Middle East & 111 & 10.4 \\
USA & 200 & 18.8 \\
UK & 105 & 9.9 \\
Canada & 59 & 5.5 \\
India & 239 & 22.4 \\
Pakistan & 202 & 19.0 \\
Other & 149 & 14.0 \\
Total & 1065 & 100.0 \\
\hline
\end{tabular}

retweets. We noted that articles from the USA, Canada and India consistently referred to WTS as 'hookah', those from the UK and Pakistan as 'shisha/sheesha', and from the Middle East as 'narghile' (the latter with a wide variation in spelling).

Over one in three (36.8\%) news articles cited scientific research. The most commonly cited publications were from $\mathrm{WHO}^{7}(32.9 \%)$ and the British Heart Foundation $(\mathrm{BHF})^{26}$ $(12.8 \%)$. The most frequently cited peer-reviewed papers were two WTS prevalence studies by Sutfin et $a l^{27}$ (2.8\% of total) and Fielder et $a l^{28}$ (1.6\% of total) and one study comparing the respiratory symptoms between WTS and cigarette smokers by Boskabady et $a l^{4}$ (2.2\% of total). These publications were partly responsible for several identified peaks when we assessed frequency trends over time (figure 1). The largest and the most sustained peaks were those coinciding with events related to health bodies (WHO and BHF) as opposed to peer-reviewed papers or other news.

Table 2 shows the top 10 key health themes in identified news articles with a country-by-country breakdown (extended table shown in online supplementary appendix 3). The mean number of health themes mentioned per news article was $3.5 \pm 3.2$ (range 1-22); $10.8 \%$ of all news articles contained positive (ie, incorrect) health themes (smoke is filtered: $3.8 \%$; less harmful than cigarettes: $2.7 \%$; herbal waterpipe is safe: $2.3 \%)$.

Additionally, over one in five articles $(22.9 \%)$ included a statement about a CWE, of which $81.2 \%$ (18.6\% in total) compared one WTS session to 100 cigarettes or more in the absence of mentioning any particular comparative parameter, such as chemical composition or volume of smoke produced (herein known as 'CWE $\geq 100$ cigarettes') (eg, 'One hour on a shisha same as 100 fags', The Sun, UK, http://tinyurl.com/6loypcp). All other CWE statements used volume of smoke or chemical composition comparisons and fell within acceptable ranges as identified by our earlier literature review. It was on news articles with a $C W E \geq 100$ cigarettes that we performed logistic regression. Website popularity was not a significant predictor in our logistic regression model. Compared with the Middle East, the odds of news articles containing a CWE $\geq 100$ cigarettes were five times higher for the USA and the UK (USA OR 4.97, 95\% CI 2.53 to $9.75, \mathrm{p}<0.001$; UK OR 5.47, 95\% CI 2.63 to 11.39 , $\mathrm{p}<0.001$ ); Canada and Pakistan were also significant predictors, as were news articles citing scientific research (table 3 ). Of the articles mentioning a CWE $\geq 100$ cigarettes, $12.5 \%$ sourced a reference for this statistic; all cited the 2005 WHO report on waterpipe smoking. ${ }^{7}$

Half $(51.4 \%)$ of the positive health themes in news articles were stated by the journalist, a third (34.9\%) were from commercial waterpipe cafe owners and the remainder were from healthcare professionals, politicians or waterpipe smokers. In comparison, most $(58.1 \%)$ of ' $\mathrm{CWE} \geq 100$ cigarette statements' were by a journalist, one-quarter $(25.3 \%)$ by healthcare professionals and $14.6 \%$ by a politician.

\section{DISCUSSION}

\section{Main results}

This study gives insight into the media reporting of WTS across countries in the online news domain. The 'Google Alerts' service provided a large volume of health-related articles which

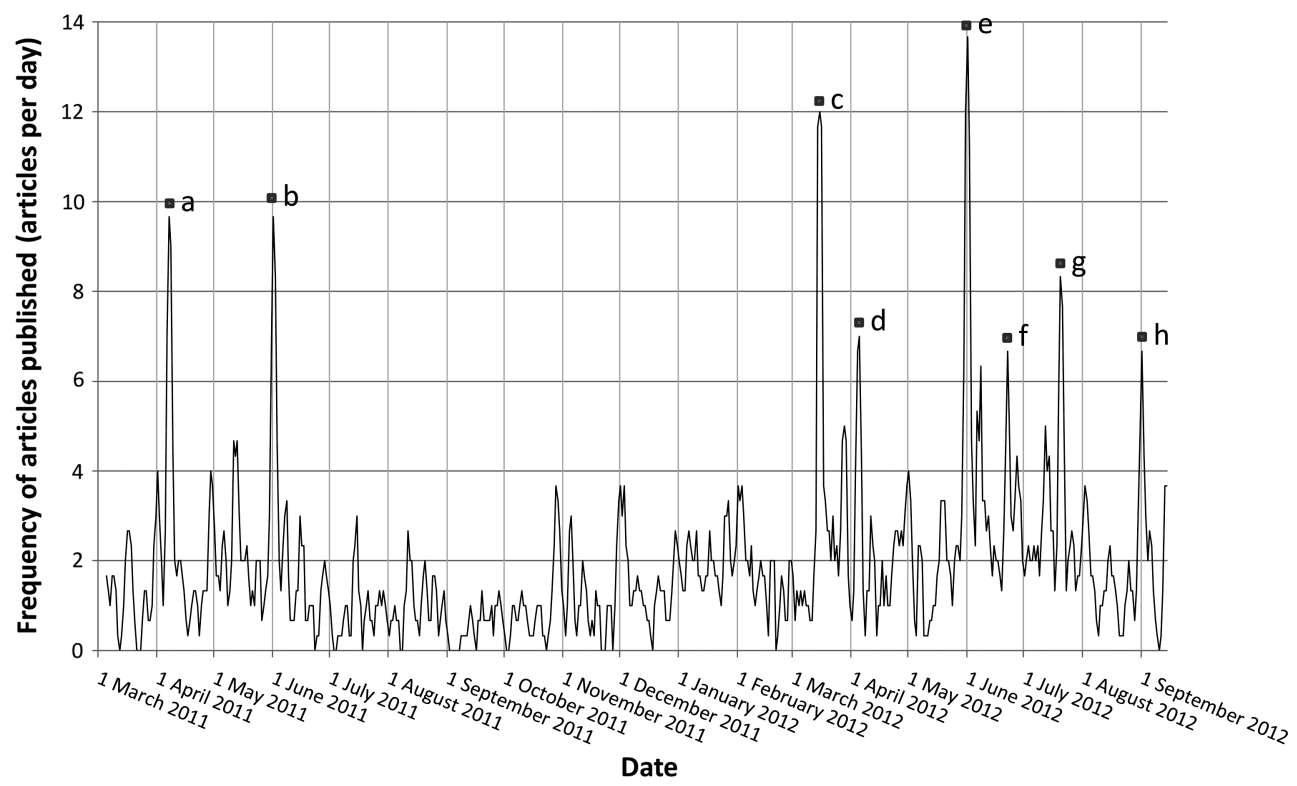

Figure 1 Frequency of news articles published (3-day moving average). (a) Prevalence study: Sutfin et al; (b) Media coverage on World No Tobacco Day; (c) Media coverage on No Smoking Day; (d) Football star from English Premier League vindicated after being photographed smoking waterpipe; (e) Media coverage on World No Tobacco Day; (f) Proposed waterpipe public smoking ban in Ottowa; (g) Prevalence study: Fielder et al; (h) Health effects study: Boskabady et al. 
Table 2 Top 10 health themes mentioned by country/region $(\mathrm{N}=2831)$

\begin{tabular}{|c|c|c|c|c|c|c|c|c|c|c|c|c|c|c|c|c|}
\hline \multirow[b]{2}{*}{ Health information } & \multicolumn{2}{|c|}{ Total } & \multicolumn{2}{|c|}{ MID E } & \multicolumn{2}{|c|}{ USA } & \multicolumn{2}{|l|}{ UK } & \multicolumn{2}{|c|}{ CAN } & \multicolumn{2}{|l|}{ IND } & \multicolumn{2}{|l|}{ PAK } & \multicolumn{2}{|c|}{ ОТн } \\
\hline & N & $\%$ & N & $\%$ & $\mathbf{N}$ & $\%$ & N & $\%$ & $\mathbf{N}$ & $\%$ & N & $\%$ & $\mathbf{N}$ & $\%$ & N & $\%$ \\
\hline 1. Contains tobacco & 709 & 66.6 & 49 & 44.1 & 177 & 88.5 & 83 & 79.0 & 35 & 59.3 & 157 & 65.7 & 102 & 50.5 & 106 & 71.1 \\
\hline 2. As bad/worse than cigarettes & 524 & 49.2 & 54 & 48.6 & 112 & 56.0 & 54 & 51.4 & 28 & 47.5 & 92 & 38.5 & 100 & 49.5 & 84 & 56.4 \\
\hline 3. Contains nicotine/is addictive & 355 & 33.3 & 32 & 28.8 & 70 & 35.0 & 15 & 14.3 & 8 & 13.6 & 130 & 54.4 & 52 & 25.7 & 48 & 32.2 \\
\hline 4. Causes cancer/produces carcinogens & 229 & 21.5 & 17 & 15.3 & 63 & 31.5 & 26 & 24.8 & 7 & 11.9 & 33 & 13.8 & 42 & 20.8 & 41 & 27.5 \\
\hline 5. Is the same as 100 cigarettes & 198 & 18.6 & 19 & 17.1 & 45 & 22.5 & 17 & 16.2 & 12 & 20.3 & 38 & 15.9 & 38 & 18.8 & 29 & 19.5 \\
\hline 5. Contains carbon monoxide & 198 & 18.6 & 18 & 16.2 & 58 & 29.0 & 23 & 21.9 & 8 & 13.6 & 22 & 9.2 & 23 & 11.4 & 46 & 30.9 \\
\hline 7. Can cause respiratory illness & 191 & 17.9 & 14 & 12.6 & 40 & 20.0 & 18 & 17.1 & 5 & 8.5 & 40 & 16.7 & 39 & 19.3 & 35 & 23.5 \\
\hline 8. Incorrect belief that smoke is filtered & 149 & 14.0 & 11 & 9.9 & 23 & 11.5 & 11 & 10.5 & 10 & 16.9 & 31 & 13.0 & 28 & 13.9 & 35 & 23.5 \\
\hline 9. Can cause heart disease & 142 & 13.3 & 12 & 10.8 & 34 & 17.0 & 22 & 21.0 & 3 & 5.1 & 16 & 6.7 & 26 & 12.9 & 29 & 19.5 \\
\hline 10. Dangers of secondhand smoke & 136 & 12.8 & 21 & 18.9 & 29 & 14.5 & 12 & 11.4 & 14 & 23.7 & 8 & 3.3 & 30 & 14.9 & 22 & 14.8 \\
\hline
\end{tabular}

CAN, Canada; IND, India; MID E, Middle East; OTH, Other; PAK, Pakistan.

we identified as a useful resource to keep updated with worldwide current affairs. Almost all articles had social media features available, allowing for a rapid response of news by its readers.

The data presented here on the type of health information mentioned in each news article by country can form the basis for future health awareness campaigns. For example, it appears that media reports neglect mentioning the presence of 'tar' $(9.7 \%)$ or the risks of secondhand smoke $(12.8 \%)$ but not so much the presence of nicotine (33.3\%) and tobacco (66.6\%). This is important as secondhand smoke is the key health theme in newspapers' cigarette coverage, ${ }^{29}$ but not a finding from this study. Different countries/regions focused on specific WTS health themes. For example, the Middle East and Canada mentioned the dangers of secondhand smoke more than other countries, which may be due to current legislation banning WTS in public areas of part of the country/region. India mentioned the nicotine content of waterpipes more than other countries and this may be due to a wider crackdown on nicotine-containing products as described by some news articles. The UK mentioned the effect of waterpipes on the heart more than other countries; this may be because the BHF was a key driver in promoting UK WTS media awareness.

Table 3 Predictors of articles mentioning cigarette-waterpipe equivalence $\geq 100$ cigarettes

\begin{tabular}{ll}
\hline Variable & Adjusted OR (95\% CI) \\
\hline $\begin{array}{l}\text { Country/region source } \\
\text { Middle East }\end{array}$ & - \\
USA & $4.97(2.53 \text { to } 9.75)^{*}$ \\
UK & $5.47(2.63 \text { to } 11.39)^{*}$ \\
Canada & $2.40(1.01 \text { to } 5.71)^{*}$ \\
India & $1.83(0.90$ to 3.73$)$ \\
Pakistan & $3.41(1.73 \text { to } 6.73)^{*}$ \\
Other & $4.83(2.40 \text { to } 9.72)^{*}$ \\
Website rank & \\
100000+ & - \\
10000-99999 & $1.07(0.73$ to 1.57$)$ \\
1000-9999 & $1.00(0.67$ to 1.49$)$ \\
1-999 & $1.15(0.67$ to 1.98$)$ \\
Mentions academic research & \\
No & - \\
Yes & $1.68(1.27 \text { to } 2.23)^{*}$ \\
\hline * $\mathrm{p}<0.001 ; ~ * * p<0.05$. &
\end{tabular}

Another major finding is the level of confusion stemming from numerical comparisons between WTS and cigarette smoking, stemming from the 2005 WHO report on WTS. Articles from the Middle East were less likely to mention a CWE $\geq 100$ cigarettes compared with other countries. This finding may be due to increased research and legislation in this region (eg, banning of cafes in residential areas, presence of health warnings on packaging; topics all mentioned in Middle Eastern news articles) and hence increased educational awareness of WTS among those who speak to the media. This may explain why countries such as the UK, the USA and Canada, who have relatively little WTS legislation, may be prone to such statements. One fact that does not support this argument is the high mention of a CWE $\geq 100$ cigarettes in India where legislation has been implemented. Regardless, the fact that over a fifth of articles mentioned some form of CWE (accurate or otherwise) may require a consensus between researchers to create such a figure for ease of public interpretation.

The 2005 WHO report on WTS was the main source of scientific information for online news articles and the only source of scientific information for news articles mentioning a $C W E \geq 100$ cigarettes. This is a serious misunderstanding of an accurate and landmark report on WTS, where its conclusions compared the volume of smoke produced by one session of WTS with the volume of smoke produced by 100 cigarettes. Since this WHO report, and as detailed in our earlier literature review, the volume of smoke produced by one waterpipe session may equate to the volume of smoke produced by 50-250 cigarettes. $^{21} 25$ In addition to the isolated literature reports mentioning a CWE $\geq 100$ cigarettes, ${ }^{12}{ }^{13}$ our findings suggest this 'catchy statistic' has extended to journalists and politicians, so it is imperative to only use such a comparison when referring solely to volume of smoke. Indeed, even with correct wording, its future use in public health campaigns is certainly questionable as it has caused confusion in the media. Indeed, it appears that in $22.9 \%$ of online news articles, numerically benchmarking the harms of waterpipes against those of cigarettes plays a role in understanding the health effects of waterpipes, and it is likely that the general public also perceive WTS in relation to the harm from a cigarette.

To our knowledge, this is the first study to perform a content analysis on news articles on WTS in the online domain. Using a robust and systematic methodology, it provides information about media behaviour in countries or regions that produce the greatest number of news articles on WTS. This can enhance the quality of future educational campaigns if public health agents 
are able to identify health themes which are largely neglected in their country's media and including them in future health promotion material.

A key limitation of this study is that it does not address outcomes, such as effect on knowledge, attitudes and beliefs of waterpipe smoking in the general public. Another limitation includes the lack of a validated methodology to measure popularity of a news website. Additionally, some news websites only transiently display their news articles, resulting in incorrect/expired URL links after several months. Had articles other than those in the English language been included in this analysis, it would have made for more robust inter-country comparisons. Finally, we used 'original' articles as part of the selection process (ie, the earliest publication date if duplicate articles were present), however the frequency of article duplication on other websites may in fact have been a better reflection of article popularity/ newsworthiness, but we did not take this weighting into account.

Despite these limitations, this study identified a potential need for simple, evidence-based WTS education among public health agents, policymakers, health journalists and community workers, addressing the confusion surrounding numerical comparisons between WTS and cigarette smoking. Half of the high publication frequency peaks of news articles we observed were due to intentional press releases about WTS (on occasions such as No Smoking Day and World No Tobacco Day). It is concerning that one in four incorrect statements about a CWE $\geq 100$ cigarettes were made by healthcare professionals or organisations.

Using simple online content retrieval programs such as 'Google Alerts' may facilitate greater understanding of the media's stance on contentious issues. Such retrieval programs can be an effective method to maintain awareness of current issues. Health themes related to WTS varied across countries and regions and a CWE $\geq 100$ cigarettes statement was commonplace. Tobacco control advocates should use the media to correct areas of confusion in the future.

\section{What is already known on this subject?}

- There is a widespread belief that waterpipe tobacco smoking (WTS) is a safer alternative to cigarettes. Although there has been a heightened media interest in WTS in recent years, no study has examined how WTS is portrayed in the news media.

\section{What this study adds?}

- This study is the first to highlight the common use of numerical cigarette-waterpipe equivalence (CWE) to relay the health effects of WTS. This study shows that a key academic source (2005 WHO report) can be misinterpreted by journalists and public health agents. Public health agents who interact with the media need further education to ensure they interpret numerical CWE correctly.

Contributors $M J, M A, A B$ and $S J$ designed the study. $M A$ and $A B$ abstracted data. EAA, MJ and SJ conducted statistical analysis. MJ wrote the first draft of the manuscript, which was edited by other authors. All authors read and approved the final manuscript.
Competing interests None.

Provenance and peer review Not commissioned; externally peer reviewed.

\section{REFERENCES}

1 AkI EA, Gunukula SK, Aleem S, et al. The prevalence of waterpipe tobacco smoking among the general and specific populations: a systematic review. BMC Public Health 2011;11:244.

2 Raad D, Gaddam S, Schunemann HJ, et al. Effects of water-pipe smoking on lung function: a systematic review and meta-analysis. Chest 2011;139:764-74.

3 AkI EA, Gaddam S, Gunukula SK, et al. The effects of waterpipe tobacco smoking on health outcomes: a systematic review. Int J Epidemiol 2010;39:834-57.

4 Boskabady $\mathrm{MH}$, Farhang L, Mahmodinia M, et al. Comparison of pulmonary function and respiratory symptoms in water pipe and cigarette smokers. Respirology 2012:17:950-6.

5 Smith JR, Novotny TE, Edland SD, et al. Determinants of Hookah use among high school students. Nicotine Tob Res 2011:13:565-72.

6 Smith-Simone S, Maziak W, Ward K, et al. Waterpipe tobacco smoking: knowledge, attitudes, beliefs, and behavior in two U.S. samples. Nicotine Tob Res 2008; 10:393-8

7 WHO. Water-pipe tobacco smoking: health effects, research needs and recommended actions by regulators. Geneva, Switzerland: World Health Organization, 2005.

8 WHO. World health organisation framework convention on tobacco control. Geneva, Switzerland: World Health Organization, 2003.

9 Nakkash R, Khalil J. Health warning labelling practices on narghile (shisha, hookah) waterpipe tobacco products and related accessories. Tob Control 2010;19:235-9.

10 Shihadeh A. Investigation of mainstream smoke aerosol of the argileh water pipe. Food Chem Toxicol 2003;41:143-52.

11 Maziak W, Eissenberg T, Ward KD. Patterns of waterpipe use and dependence: implications for intervention development. Pharmacol Biochem Behav 2005;80:173-9.

12 Masters $N$, Tutt C, Yaseen N. Waterpipe tobacco smoking and cigarette equivalence. Br J Gen Pract 2012;62:127

13 Rastam S, Li FM, Fouad FM, et al. Water pipe smoking and human oral cancers. Med Hypotheses 2010;74:457-9.

14 ASH. 'Shisha 200 times worse than a cigarette', say Midde East expert. London: Action on Smoking and Health, 2007.

15 Pierce JP, Gilpin EA. News media coverage of smoking and health is associated with changes in population rates of smoking cessation but not initiation. Tob Control 2001;10:145-53.

16 Dedobbeleer N, Beland F. Gender and the social context of smoking behaviour. Soc Sci Med 2004;58:1-12.

17 Klegg Smith K, Wakefield MA, Terry-McElrath Y, et al. Relation between newspaper coverage of tobacco issues and smoking attitudes and behaviour among American teens. Tob Control 2012;17:17-24.

18 Laugesen K, Meads C. Advertising, price, income and publicity effects on weekly cigarette sales in New Zealand supermarkets. Br J Addiction 1991;86:83-9.

19 Wakefield MA, Brennan E, Durkin SJ, et al. Still a burning issue: trends in the volume, content and population reach of newspaper coverage about tobacco issues. Criti Pub Health 2011;21:313-25.

20 Landis JR, Koch GG. The measurement of observer agreement for categorical data. Biometrics 1977;33:159-74.

21 Katurji M, Daher N, Sheheitli $\mathrm{H}$, et al. Direct measurement of toxicants inhaled by water pipe users in the natural environment using a real-time in situ sampling technique. Inhal Toxicol 2010;22:1101-9.

22 Shihadeh A, Salman R, Jaroudi E, et al. Does switching to a tobacco-free waterpipe product reduce toxicant intake? A crossover study comparing $\mathrm{CO}, \mathrm{NO}, \mathrm{PAH}$, volatile aldehydes, 'tar' and nicotine yields. Food Chem Toxicol 2012;50:1494-8.

23 Shihadeh A, Saleh R. Polycyclic aromatic hydrocarbons, carbon monoxide, 'tar', and nicotine in the mainstream smoke aerosol of the narghile water pipe. Food Chem Toxicol 2005;43:655-61.

24 Neergaard J, Singh P, Job J, et al. Waterpipe smoking and nicotine exposure: a review of the current evidence. Nicotine Tob Res 2007;9:987-94.

25 Eissenberg T, Shihadeh A. Waterpipe tobacco and cigarette smoking. Direct comparison of toxicant exposure. Am J Prev Med 2009;37:518-23.

26 BHF. Rise in 'shisha bars' prompts warning on dangers of waterpipe smoking. London: British Heart Foundation, 2012.

27 Sutfin EL, McCoy TP, Reboussin BA, et al. Prevalence and correlates of waterpipe tobacco smoking by college students in North Carolina. Drug Alcohol Depend 2011;115:131-6

28 Fielder RL, Carey KB, Carey MP. Prevalence, frequency, and initiation of hookah tobacco smoking among first-year female college students: a one-year longitudinal study. Addict Behav 2012;37:221-4.

29 Durrant R, Wakefield M, McLeod K, et al. Tobacco in the news: an analysis of newspaper coverage of tobacco issues in Australia, 2001. Tob Control 2003;12 (suppl 2):ii75-81. 